\title{
Mental Bases of Social Solidarity
}

\author{
Nikolay N. Gubanov \\ Bauman Moscow State Technical University \\ 5/1, 2nd Baumanskaya St. \\ Moscow, Russia 105005 \\ E-mail: gubanovnn@mail.ru
}

\author{
Nikolay I. Gubanov \\ Tyumen State Medical University \\ 54, Odesskaya St. \\ Tyumen, Russia 625023 \\ E-mail: gubanov48@mail.ru
}

\begin{abstract}
Four pairs of mental features are singled out and characterized: constructive similarities, destructive similarities, constructive differences, destructive differences. Constructive similarities and differences cause the integration of the society, while destructive similarities and differences lead to its disintegration. To increase the attraction between any individual or group subjects, we must increase constructive similarities and constructive differences, reduce destructive similarities and destructive differences. The most important tendency for mankind to form general civilization solidarity on the basis of constructive similarity was discovered and described.
\end{abstract}

Keywords-solidarity; constructive similarities; constructive differences; destructive similarities; destructive differences; $E$. Durkheim; intensity of attraction-repulsion

\section{INTRODUCTION}

In the last two decades, when describing and explaining various aspects of the functioning of society, the category of "stamp of mind", or "mentality" has been widely used. It opens new perspectives in social cognition. Under mentality we mean a set of socio-psychological characteristics of a person or a social group that determines the specific nature of their thinking, perception of the world and activity [1]. The purpose of this paper is to use this category to develop an urgent problem of social solidarity. Let us note that one of the functions of the mentality is the formation of social solidarity, which, as shown by E. Durkheim, is the main force that unites the society, creating a social whole, ensuring its stability and sustainable development [2]. The difficulty in ensuring public solidarity is that the mentality of the society of even one though quite numerous community, for example, nation, is heterogeneous. To understand the conditions for the implementation of solidarity, which is able to firmly integrate this community, it is necessary to reveal the mental similarities and differences of various groups including into it.

\section{CONSTRUCTIVE AND DESTRUCTIVE MENTAL FEATURES}

The analysis of the relations between people allows us to assume that affection and cooperation can arise both on the basis of similarities and on the basis of differences [3]. However, not all differences can lead to attraction. To make the differences attract, they should not be mutually exclusive, but mutually supportive, complementary. Usually a certain mental difference causes affection if one person has a certain quality that no other person has, but the latter would like to possess it. Let us call such mutually complementary dissimilarities, which cause mutual attraction, constructive. Durkheim notes that a theorist with a subtle analytical mind often has a special inclination to practical people, common sense, quick intuitions; a timid person has an affection for courageous and resolute people; a weak person look toward strong and determined and vice versa. No matter how much we are gifted, we constantly lack something, and the best of us feel our imperfection. That is why we are looking for our missing qualities in our friends: by connecting with them, we somehow become involved in their nature and feel more perfect. In this way, small associations of friends are created, where everyone has a role, in accordance with their character, where there is a real exchange of services. One patronizes, the other one comforts, the third one advises, the fourth one fulfills, and it is this division of functions, or the division of labor, that causes a relationship of friendship [4].

The differences between the artistic and mental types of personality identified by I.P. Pavlov are constructive in the society. People of the first type are dominated by figurative thinking, and they are successfully engaged in art. And representatives of the second type are dominated by conceptual thinking, and they are successful in science [5]. Culture and society as a whole benefit from the existence of such mental differences between people, because it is a condition for the harmonious development of all branches of culture. K.G. Jung describes the types of "introvert" and "extrovert", he points out that mainly those people who belong to different types get married, they do it unconsciously - to complement each other. The reflective introvert nature encourages the person to constantly meditate or collect his or her thoughts before doing something. Their timidity before the objects and distrust of them cause hesitation. An extrovert, on the contrary, has a positive attitude toward things. New situations attract him. To find out something unknown, he or she is even ready to plunge into it without looking back. As a rule, they act first and only then think about it. These two types are therefore created for symbiosis. One takes care of the thinking, and the other takes the initiative and practical actions. Therefore, the marriage between representatives of these two different types can be ideal [6]. 
Thus, we come to the conclusion that some differences, which we have designated as constructive, lead to the attraction of subjects, and some, we call them destructive, lead to repulsion. Apparently, the same applies to mental similarities. Some similarities attract each other, let us call them constructive, and some repel, let us call them destructive. As examples of constructive similarities we can note common interests, values-based orientations, goals, ideals, experiences, and much more. Destructive similarities include aggressiveness, irritability, intolerance of criticism, deceit, greed. In the society, non-constructive similarity arises where elements are redundant in terms of implementing a function of the system.

Bearing mind the ideas mentioned above, we can conclude that, when the subjects interact, there are four pairs of mental features: constructive similarities, destructive similarities, constructive differences, destructive differences. Constructive similarities and differences cause the integration of the society, and destructive similarities and differences cause its disintegration. If we use a system of education or organizational measures to replace one mental feature which enters into a destructive difference, of one of the subjects with the opposite one, then we get a constructive similarity at this point [7]. For example, if two subjects have opposite goals, then if we replace a goal of one of them, we get a constructive similarity of goals. Repulsion is changed to gravity. If we replace one mental feature entering into a constructive difference, of one of the subjects with the opposite, then we, on the contrary, weaken the attraction between the subjects. In this case, we change the constructive difference to the destructive similarity. For example, if the complementary difference was manifested in the fact that a timid person stained after a courageous one, and the latter suddenly lost courage due to some circumstances and became timid too, then the attraction of the first to the second will weaken or even become contemptible. [8] Hence, in order to increase the attraction between any actors, we must increase constructive similarities and constructive differences, reduce destructive similarities and destructive differences.

Each pair of mental characteristics that determine the degree of social solidarity as a result of summation has its own attraction-repulsion intensity. The deeper and, therefore, more stable are the interacting mental features, the greater the intensity of attraction or repulsion will be given by this pair and, consequently, the more it integrates or disintegrates upon contact of these subjects. J. Habermas notes that the choice regarding trivial or weak preferences does not require justification; no one is obliged to report to himself or others, why he or she prefers this brand of a car or the given style of a pullover. On the contrary, by strong preferences we call the assessments that affect not only casual predispositions and inclinations, but self-awareness of the individual, lifestyle, character; they are inseparable from the identity of ourselves [9].

\section{THE LAW OF MEANING OF MENTAL}

\section{DIFFERENCES AND SIMILARITIES OF SOCIAL GROUPS}

Due to the fact that destructive similarities and destructive differences with a fruitful existential dialogue have the potential for transition to factors of solidarity strengthening (constructive similarities and constructive differences), we believe that in society, in order to ensure sustainable development, there must exist some reasonable proportion, or measure, between all four pairs of mental features to ensure the necessary unity and diversity of the social system and its higher adaptive abilities. The relationship described above can be called as the law of the measure of mental difference and the similarity of social groups as a condition of social solidarity and progress. According to this law, constructive mental similarities should ensure the spiritual unity of the given society, constructive differences should serve as a stimulus for the production of cultural innovations, destructive differences and the contradictions generated by them are resolved in the course of existential dialogue, and non-constructive and destructive similarities are tolerated and subsequently transformed into constructive ones. The balance of integration must prevail over the balance of disintegration. Even destructive differences, if they are moderate, can become a factor of progress when the spiritual and ruling elite create and implement measures to curb the negative social phenomena associated with these differences. At the same time, the society accumulates experience of coping with destructive differences, and some future social cataclysms, exacerbating destructive differences, will not take this society by surprise [10].

If we sum up all the above, then we can draw the conclusion: the magnitude of social solidarity represents the resultant of four mental factors of strengtheningweakening of social ties: each constructive mental similarity and constructive difference are included in the sum of total solidarity with the plus sign and multiplied by its weight factor, characterizing the intensity of attraction of this pair; each destructive similarity and destructive difference are included a minus sign and their weight coefficient, which shows the intensity of repulsion of this pair.

Durkheim introduced two types of solidarity: solidarity, arising on the basis of similarities, and solidarity arising from the division of labor, and hence from differences. He calls the first kind of solidarity the mechanical solidarity. The most vivid examples of almost hundred-per-cent mechanical solidarity can be found in primitive and archaic societies, when there is practically no difference between its members, where even women differ little from men and perform similar functions. Durkheim called the second kind of solidarity the organic solidarity. It arises as a result of the division of functions and is characterized by the fact that individuals are becoming increasingly different from each other. He drew an analogy with an organism in which there are dissimilar formations that perform specific functions. The organic solidarity, according to Durkheim, is a more cohesive force than the mechanical one, since if mechanical particles can be divided without damage to each other, organs cannot exist without each other, they exist only 
in integrity, the organism (society) being their integrity. The organic solidarity prevails in industrial, developed societies. The individual consciousness here receives an increasing specific increment to the collective consciousness, which, in turn, gradually decreases. The main indicator of the organic solidarity in society is the rules governing various activities and fixed in the restitution law.

According to Durkheim's concept, the place of the mechanical solidarity is increasingly occupied by the organic one because of the constantly ongoing process of differentiation of activity in society [11]. The mechanical solidarity is constantly decreasing in its scope, however, it is not doomed to total withering away, there is always some very general content of collective consciousness. While the mechanical solidarity is declining, the organic solidarity, thanks to the division of labor, is increasing, automatically leading society to the heyday of democracy, increasing respect for the individual, and the full protection of human rights.

We will comment on Durkheim concept from the standpoint of the methodological principles we have adopted. Let us call his mechanical solidarity as the solidarity on the basis of constructive similarities, and the organic solidarity as the solidarity on the basis of constructive differences. Durkheim abstracts from destructive similarities and destructive differences, which are factors of the reduction of solidarity, which, however, constantly arise together with factors of strengthening solidarity. These factors, weakening solidarity, do not fall within the scope of its systematic consideration. We consider this to be a significant drawback of Durkheim concept, since the division of labor, like any other sufficiently large social phenomenon, does not lead to automatic strengthening solidarity, but, in general, to the emergence of four pairs of mental features that determine the general solidarity that will be determined by arithmetic of solidarity described above. Everything depends on how, where, under what circumstances the division of labor takes place. For example, the division of citizens into law abiding and criminals does not lead to an increase in overall solidarity. In the course of history, there are both the factors for strengthening solidarity, as well as the factors for its reduction (destructive similarities and destructive differences). There will always be a conflict between someone's interests, goals, values (destructive differences), and there will always be those who try to stand out among other similar people and feel hostile to them because of certain types of similarities (destructive similarities) [12]. The task of the ruling elite is exactly the calculation of solidarity and the choice of such reforms, legislative decisions and other regulations, so that the overall solidarity should not fall below a dangerous level, but if possible, it should increase.

\section{Changes of Mental ReCOGNitions AND DIFFERENCES IN THE SOCIETY}

Destructive differences that have not been the subject of Durkheim research can occur not only between social groups within the country, but also between countries [13]. In the scale of the planet, destructive differences between countries have caused two world wars. We do not share Durkheim's opinion that in the course of history there is a constant change of the mechanical solidarity by the organic one. In our opinion, there are constant fluctuations and a change in the proportion in which there are solidarities by constructive similarities and constructive differences. Since only these two factors strengthen solidarity, with a decrease in one of them, it is desirable to increase the second one, so as not to outweigh the factors that reduce solidarity. With the division of labor, there is a production of both the factors strengthening solidarity and the factors reducing it. According to Durkheim, with the reduction of solidarity by similarities, solidarity is growing in the division of labor (it is becoming stronger). In our opinion, everything is not so clear. The division of labor is always carried out in some external conditions, between certain specific groups. The division of labor in the context of specific historical circumstances leads to the growth of solidarity in the constructive distinction between the specializing groups but, on the other hand, to the increase in solidarity by constructive similarity within each of these communities. Thus, these two kinds of solidarity are related. Only the intensity of attraction and the speed of the formation of similarities-differences that determine these types of solidarity can be different, therefore we cannot say that both solidarities increase together in a single proportion.

In our opinion, there are no sufficient grounds for believing that in general, in any society there is always a persistent tendency to reduce the overall solidarity by constructive similarities and a constant increase in the share of solidarity by constructive differences. Examples can be given when there is an increase in solidarity by constructive similarities, and solidarity by constructive differences is reduced. For example, during the national liberation wars the solidarity of the first type sharply increases, and all the differences are erased and are no longer perceived so sharply. In the mentality of such a people, the main opposition is "ours is not ours," all other differences are perceived with less intensity. The first type of solidarity prevails in monoreligious traditional societies. Perhaps Durkheim would say that all the examples described above are also anomalous forms. But such rallying events have always occurred, and nothing points to their disappearance in the future.

There is another consideration why we cannot agree with Durkheim's view that solidarity in the division of labor tends to be a constant historical increase. P. Sorokin according to the vast historical data showed that differentiation in society cannot be infinite. For example, vertical differentiation (stratification) is the subject to fluctuation only over time. In the change in the history of mankind he found and examined in detail three main types of stratification - economic, political, professional.

Indicators of these types of social stratification, such as height and stratification profile, made only fluctuations in different directions without a stable single long-term trend. The trends acted only for a certain period of time, after which they were replaced by the opposite ones [14]. Thus, the growth of economic differentiation cannot continue indefinitely. As soon as the height of the social economic 
pyramid reaches a sufficiently large value, social forces come into force, which lead to the fact that several floors of the pyramid will be knocked down. In times of some revolutions, the pyramid in general can become very flat but after the emergence of a new power, other social forces begin to build new floors, stratify the society and so on until the next equating. Therefore, we believe that there is no historical basis for considering Durkheim's point of view to be justified. According to him, solidarity in the division of labor should only increase, and as it is stronger than the mechanical solidarity, hence, the degree of general solidarity in the world should only increase. Durkheim explained the existence of social conflicts in various parts of the world by the fact that solidarity in the division of labor had not yet had time to consolidate properly, so the mechanical solidarity had collapsed but the new, the organic one, had not yet arrived in time to replace it. Such a state, in which old values and norms no longer function, and new ones have not arisen yet, was called by Durkheim as the public anomy, and he suggested solving these local problems through reforms. But on the whole and in general, the division of labor itself would immediately lead sooner or later to a universal high solidarity, to the triumph of peace and cooperation throughout the world. This view was based on a small amount of historical experience and now seems naive. Without persistent and purposeful efforts of the ruling and intellectual elites all over the world, without any joint projects, without concerted action, no spontaneous process, including the division of labor, can lead to any positive result. The First World War dramatically ruined the optimistic pathos of Durkheim's sociological school. Many members of the sociological school perished in the war, the son of Durkheim, a young talented sociologist and linguist Andre, whom Durkheim considered to be the successor of his lifework, also died.

This allows us to conclude that after the emergence of the organic solidarity, solidarity on the basis of constructive similarities did not disappear but continues to exist. Only in their unity, with observance of the necessary measure, the society can be stable. Since the second half of the 20th century, there has been a steady trend of strengthening solidarity on the basis of constructive similarities.

Finally, the third position of Durkheim, which, in our opinion, has not been confirmed by historical experience, is that solidarity in the division of labor (in our opinion, in terms of constructive differences) is stronger than solidarity by similarities (in our opinion, by constructive similarities). We would note here that both types of solidarity complement each other and there are always processes of strengthening and weakening both types of solidarity. The parameters of solidarity depend on many factors, including random external factors, as well as on the specificity and state of the interacting entities. When Durkheim gives an example that in some primitive societies the connection between their members was not very strong, it only reflects the state of general solidarity that existed at that time in those particular societies and nothing more. Constructive mental similarities could lead to deeds and self-sacrifice, when they reached the necessary intensities, but could cause simple sympathy if the intensity was weak, or even remain indifferent if the intensity of this pair of mental similarities was approaching zero. The same is true in respect to constructive differences.

The industrial and economic development of societies, according to Durkheim, leads to a gradual blurring of group differences due to the growth of individual differences within each of the groups. The groups are less and less different, and the individuals within the groups differ from each other more and more. We would not join this Durkheim view. There is not only an unequivocal movement towards a constant increase in the differences between individuals around the world. The modern economic system, in which the international monopolies play a huge role, in some cases is aimed at creating similarities in values, standards and types of consumption, which they achieve through advertising, propaganda and other types of imposition. What constant increase of individual differences can we speak about?! We believe that there is only an increase in individual differences on one front, an increase in similarities in others (programmed by powerful economic monopolies, among others). And all this varies greatly from one society to another, depending on the closeness or openness of the society to the world community, on the strength of their own traditions and many other things, including the intensity and duration of the imposition and advertising. And in this case we have only fluctuations in similarities-differences in space and time, and not constant fundamental tendencies, as Durkheim thought. Ideally, it would be desirable that constructive similarities between individuals around the world should grow not on the front of standards and patterns of high consumption, but on the front of the adoption of universal values that would constitute the motivational core of a new type of mentality - the globalist mentality.

The development of democracy leads to a constant increase not only in constructive differences, as Durkheim believed, but also in some constructive mental similarities. For example, the basis of democracy itself is the principle of respect for the originality of another person. This norm is the constructive similarity for people who internalized the ways of democratic thinking [15]. True, Durkheim also noted this democratic constructive similarity, but believed that it was almost the only thing that remains common (in his terminology, constitutes the general, collective consciousness) for all individuals. In fact, this is hardly the only similarity that is called upon to provide the universal solidarity in the modern society on the basis of constructive similarities. From the middle of the 20th century, humanity has received the most powerful challenge of history in the form of global problems. This challenge generates a stable and important for humanity trend to form a common civilizational solidarity on the basis of constructive similarities. Progressive social forces around the world are constantly developing ideas about the so-called universal values, developing international law based on the acceptance or rejection of any social phenomena by the world community, a new type of mentality is being formed - a globalist mentality that will serve as a necessary condition for preventing inter-civilizational conflicts [16]. 


\section{CONCLUSION}

In conclusion, it should be noted that Durkheim developed an original concept based on the concepts of the mechanical and organic solidarity. However, the limited historical experience of the first half of the 20th century caused the incompleteness and inaccuracy of his concept. New historical experience makes it possible, first, to take into account the influence of constructive mental similarities and constructive differences on solidarity, destructive similarities and destructive differences of subjects, and secondly, to reveal the most important, life-changing for mankind tendency to strengthen the planetary solidarity on the basis of constructive similarities of civilizations, the tendency dating back to the second half of the 20th century and leading to the emergence of a globalist mentality.

\section{REFERENCES}

[1] Gubanov N.I., Gubanov N.N. The role of mentality in the development of society: sociocultural hypothesis // Vestnik slavianskikh kultur-bulletin of slavic cultures-scientific and informational journal. 2017. Vol. 43. № 1. P. 38-51.

[2] Durkheim E. The Division of Labour in Society. Trans. W. D. Halls, intro. Lewis A. Coser. New York: Free Press, 1997, pp. 39, 60, 108. [In Russ.: E. Durkheim. The Division of Labour in Society. Moscow, Nauka Publ., 1990.].

[3] Gubanov N.I., Gubanov N.N. Mentality: the nature and functioning in society // Voprosy filosofii. 2013. № 2. P. 22-32.

[4] Durkheim E. opere citato

[5] Pavlov I.P. Lectures on the work of the cerebral hemispheres // I.P. Pavlov. Complete works. Vol. IV. Moscow-Leningrad, USSR AS Publ., 1951, pp. 3-478.

[6] Jung K.G. Psychology of the Unconscious, 1916. [In Russ.: Jung K. Psychology of the Unconscious. Moscow, Canon + Publ., 2003, pp. 27-171.

[7] Oseledchik M.B., Ivlev V.Yu., Ivleva M.L. The fractal nature of implicit knowledge // Proceedings of the 3-rd International Conference on Arts, Design, and Contemporary Education. (ICADCE 2017). Advances in Social Science, Education and Humanities Research. Paris: Atlantis Press, 2017. V.144. Pp. 673-676.

[8] Lazarev F.V., Lebedev S.A. The Philosophical Reflex: Essence, Form, Types // Voprosy filosofii. 2016. № 6. P. 15-28.

[9] Habermas U. Democracy. Mind. Morality (Lectures and interviews). Moscow, Nauka Publ.. 1992.

[10] Nekhamkin V.A. A counterfactual Challenge of the Past: Ways of Negotiation // Herald of the Russian Academy of Sciences. 2017. Vol 87. Issue 2. P. 191-198. DOI: 10.1134/S1019331617020046

[11] Durkheim E. opere citato

[12] Komissarov I; Nekhamkin V. The Models of Historical Cognition: Current Status and Prospects of Development // Istoriya-Elektronnyi nauchno-obrazovatelnyu zhurnal. 2017. Vol. 8. Issue 2. DOI: $10.18254 /$ S0001779-5-1

[13] Suzdaleva T.R. Migratory processes in the context of geopolitics // Vestnik Tomskogo gosudarstvennogo universiteta-Filosofiyasotsiologiya-politologiya-Tomsk state university journal of philosophy sociology and political science. 2016. № 3 (36). P. 237244. DOI: $10.17223 / 1998863 X / 35 / 25$

[14] PA Sorokin. Social mobility. Moscow, Academia Publ., 2005.

[15] Ivlev V.Yu., Oseledchik M.B. Methodological principles for the introduction of modality categories in modern scientific cognition // Proceedings of the 3-rd International Conference on Arts, Design, and Contemporary Education. (ICADCE 2017) Advances in Social Science, Education and Humanities Research». Paris: Atlantis Press, 2017. V.144. Pp.541-545.
[16] Gubanov N.I., Gubanov N.N. Global mentality as a pre-condition of civilizations' conflict prevention // Sotsiologicheskie issledovaniya. 2011. № 4. P. 51-58. 